\title{
Diffusion Imaging of the Congenitally Thickened Corpus Callosum
}

N.K. Rollins

\begin{abstract}
SUMMARY: This report presents 4 subjects with congenital segmental callosal thickening, an uncommon malformation studied with MR imaging and DTI. Medical records were reviewed for genetic testing and neurodevelopmental status. Three subjects had profound developmental delay; 3 had seizures. MR imaging showed segmental thickening of the rostral and/or midcallosal body. Associated anomalies included polymicrogyria in 1 patient and optic hypoplasia in 1. DTI showed that the segmental thickening was due to anomalous longitudinal supracallosal fibers visually separable from the paired cingulum in 3 patients; in 1 patient, the cingulum was poorly formed. Genetic testing was negative for Fragile X syndrome. Microarray DNA analysis showed 3 copy losses (2q27.3, 3p21.31, 7q21.11) and 1 copy gain (8p11.23) in 1 patient, while testing in the other subject was negative for losses or gains. Potential explanations for the anomalous fibers include heterotopic cingulum, an enlarged indusium griseum, and aberrant callosal fibers.
\end{abstract}

ABBREVIATIONS: FA = fractional anisotropy; IG = indusium griseum; $\mathrm{M}-\mathrm{CMTC}=$ marmorata telangiectatica congenita syndrome; $\mathrm{NF}=$ neurofibromatosis

C onventional MR imaging is useful in the characterization of congenital anomalies of the corpus callosum, which are typically classified according to the pattern and extent of callosal hypoplasia. ${ }^{1,2}$ Diffusion imaging and diffusion tractography provide information about the composition of the normal corpus callosum by delineating the topography and the link between callosal microstructure and function. ${ }^{3}$ In callosal hypogenesis, diffusion imaging depicts aberrant heterotopic callosal fibers and provides insight into the topographic orientation of the Probst bundles in callosal agenesis. ${ }^{4,5}$ In contrast to callosal agenesis, which is a relatively common brain malformation, congenital anomalies in which all or a portion of the corpus callosum is thickened are uncommon and limited to a case report by MR imaging and a series by prenatal sonography. ${ }^{6,7}$ There are few reports of DTI of the thickened corpus callosum. ${ }^{8}$ This report presents 4 patients with segmental thickening of the rostral and/or midcallosal body in which DTI showed that the thickening was due to anomalous longitudinal midline fibers. The associated neurocognitive deficits suggest that the anomalous longitudinal

Received April 17, 2012; accepted after revision June 5.

From the Department of Radiology, Children's Medical Center Dallas-University of Texas Southwestern Medical Center at Dallas, Dallas, Texas.

Please address correspondence to Nancy K. Rollins, MD, Department of Radiology, Children's Medical Center Dallas-University of Texas Southwestern Medical Center at Dallas, 1935 Medical District Dr, Dallas, Texas 75235; e-mail: nancy.rollins@ childrens.com

-- Indicates open access to non-subscribers at www.ajnr.org

http://dx.doi.org/10.3174/ajnr.A3245 fibers may be indicative of a more widespread disorder of axonal development, which may escape detection with conventional MR imaging.

\section{MATERIALS AND METHODS}

This study was approved by the institutional review board. Given the retrospective nature, informed parental consent was not obtained. During a 10-year period, 4 subjects were identified with focal or diffuse thickening of the corpus callosum by conventional MR imaging, who also underwent DTI and diffusion tractography. Conventional MR imaging included multiplanar T1, T2 FLAIR, and T2 fast spin-echo sequences at $1.5 \mathrm{~T}$ or $3 \mathrm{~T}$. The corpus callosum was defined as thickened on the midsagittal T1 image when the thickness of the callosal body exceeded the thickness of the splenium and genu; the callosal thickness was measured by using electronic calipers on the MR imaging operator console. DTI was performed by using single-shot echo-planar imaging with the following parameters: TR/TE, 7000-9000/84-102 ms; $128 \times 128$ matrix; $2-\mathrm{mm}^{3}$ voxel size; 2 -mm section thickness; no gap. Diffusion gradients were applied in 30 noncollinear directions with $b=1000 \mathrm{~s} / \mathrm{mm}^{2}$. There were $2-3$ acquisitions covering the whole head. Scanning time for DTI ranged from 12 to 15 minutes. Eddy current correction, image registration, and motion correction were performed on the MR imaging operator console before transfer of diffusion data to a proprietary workstation (Extended WorkSpace; Philips Healthcare, Best, the Netherlands). A pediatric neuroradiologist, with $>20$ years of experience and extensive experience with diffusion tractography, manually placed 
Demographics and anomalies associated with thickened corpus callosum

\begin{tabular}{cllclll} 
Case & \multicolumn{1}{c}{ Age/Sex } & \multicolumn{1}{c}{$\begin{array}{c}\text { Indication } \\
\text { for MRI }\end{array}$} & $\begin{array}{c}\text { Maximum Callosal } \\
\text { Thickness }\end{array}$ & \multicolumn{1}{c}{ Fornices } & \multicolumn{1}{c}{ Cingulum } & \multicolumn{1}{c}{$\begin{array}{c}\text { Associated } \\
\text { Abnormalities }\end{array}$} \\
\hline 1 & $25 \mathrm{mo} /$ Male & Seizures, DD & $14.2 \mathrm{~mm}$ & Thickened & Formed bilaterally & None \\
2 & $20 \mathrm{mo} /$ Female & Seizures, mild DD & $8.7 \mathrm{~mm}$ & Atrophic right & Incompletely formed left & Acquired right MTS \\
3 & $18 \mathrm{mo} /$ Male & Seizures, DD & $9.9 \mathrm{~mm}$ & Normal & Formed bilaterally & PMG, PGH \\
4 & $12 \mathrm{yr} /$ Male & $\begin{array}{c}\text { Autism, DD, decreased } \\
\text { vision, seizures }\end{array}$ & $9.2 \mathrm{~mm}$ & Normal & Hypoplastic & Optic pathway hypoplasia \\
& & & & & \\
\hline
\end{tabular}

Note:-DD indicates developmental delay; PMG, polymicrogyria; PGH, periventricular gray matter heterotopia; MTS, mesial temporal sclerosis.

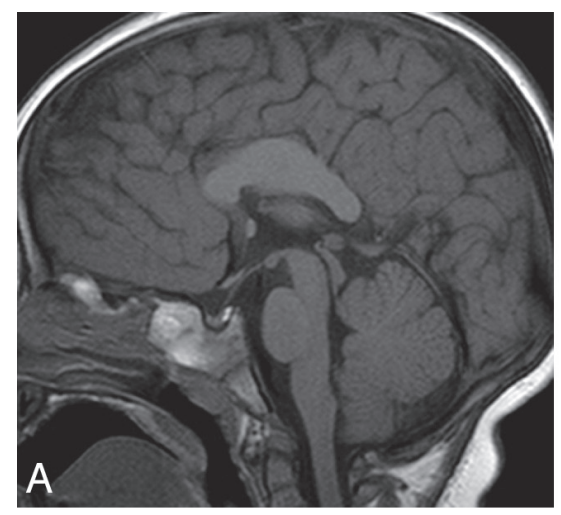

B
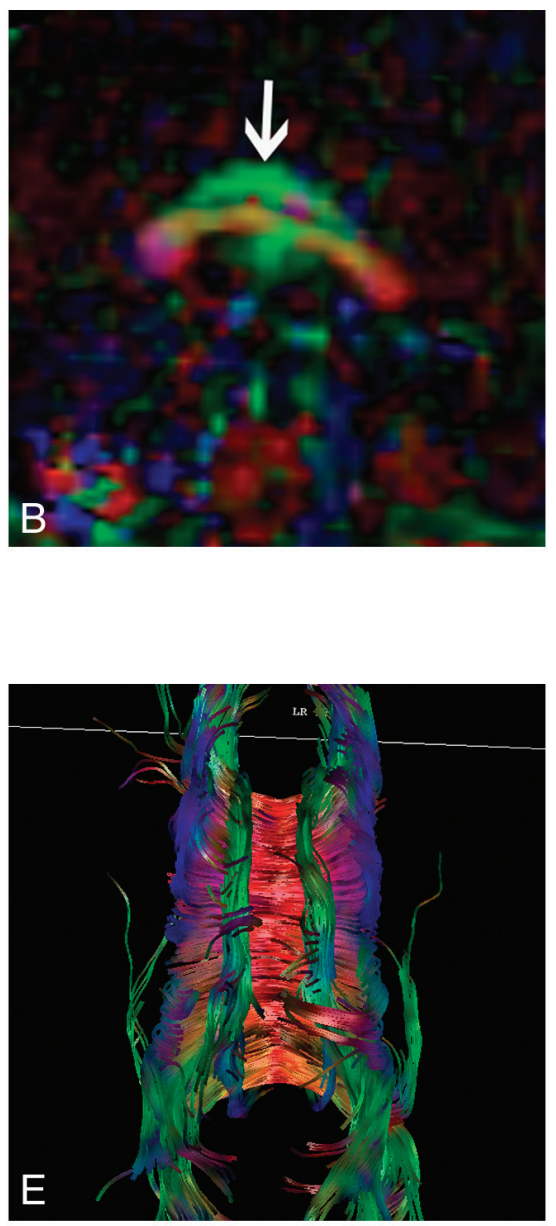
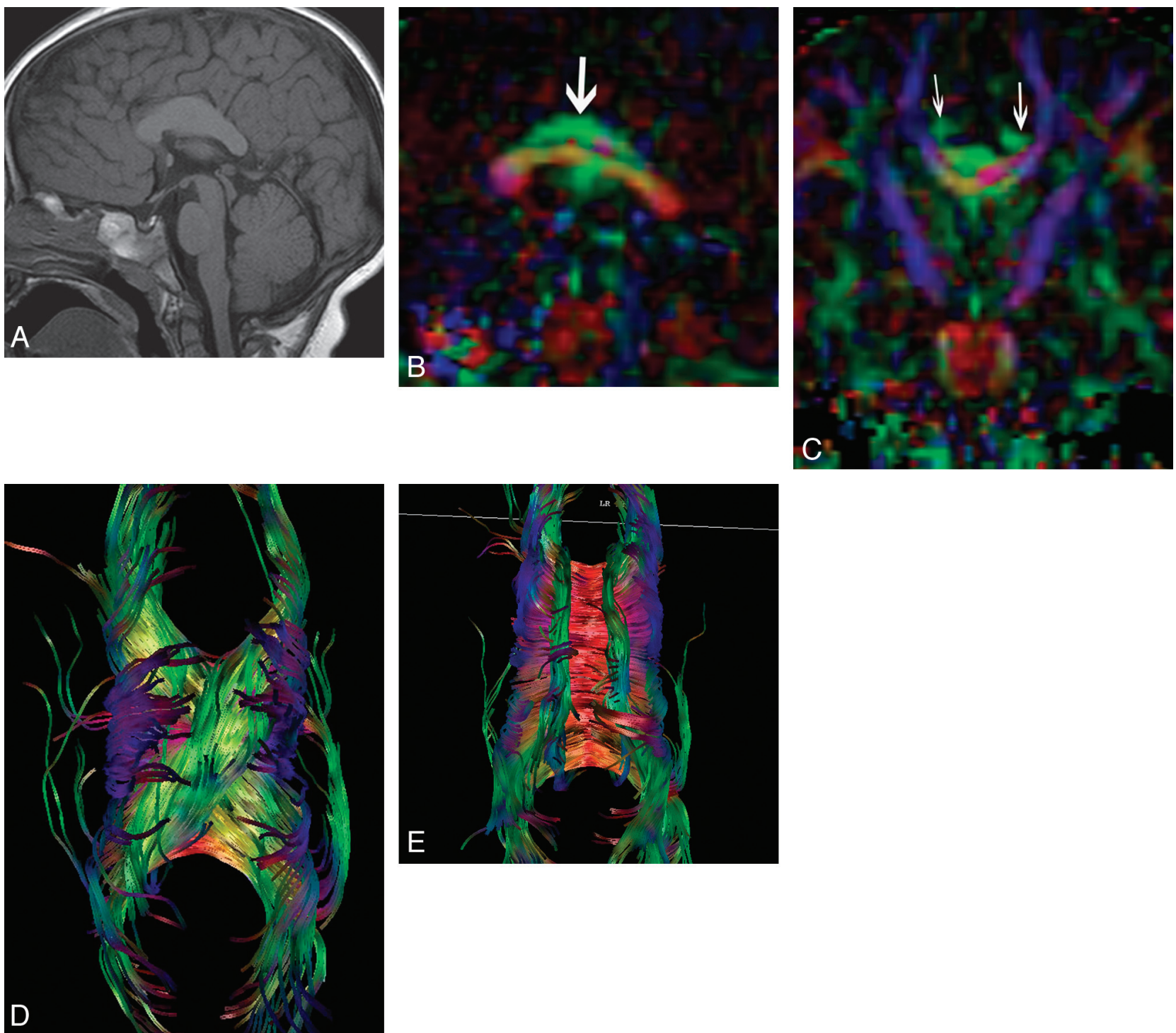

FIG 1. A 2-year-old boy with pervasive developmental delay. A, Midsagittal $T 1$ image shows marked thickening of the midcallosal body (14.2 mm) with subjective shortening of the callosal length. B, Midsagittal directionally encoded color map. The arrow indicates longitudinal supracallosal fibers, which account for the callosal thickening. Longitudinal fibers are not normally seen in the midline; like association fibers, the normal cingulum does not cross the midline. C, Coronal color map. The large midline longitudinal fibers are separate from the paired cingulum (arrows). $D$, Top-down projection of the supracallosal fibers on diffusion tractography. E, Axial top-down tractography from a healthy subject for comparison. The cingulum is seen as parasagittal rather than midline.

regions of interest on the directionally encoded color maps, encompassing the corpus callosum and supracallosal fibers so that the resultant 3D renderings included the entire corpus callosum and the cingulum, which form the outer limbic arch. Diffusion tractography was performed by using fiber association by continuous tractography, in which tracts were seeded from all voxels in the corpus callosum and supracallosal fibers had FA $>0.15$ and a turning angle of $<70^{\circ}$. Because the diffusion image data had not been acquired by using high-angular-resolution diffusion and given the insensitivity of DTI to intravoxel crossing fibers, fibers were not further segmented according to projections to specific areas of the brain. 


\section{CASE STUDIES}

Patient 1 was a 2 -year-old boy with pervasive developmental delay, hypotonia, and generalized seizures since infancy, born full-term after an uncomplicated delivery to nonconsanguineous parents. There were no dysmorphic features, and head circumference was normal. Genetic testing limited to Fragile $\mathrm{X}$ syndrome was normal (Table). MR imaging showed that the corpus callosum was shortened in an anteroposterior dimension; the midcallosal body measured $14.2 \mathrm{~mm}$ in the region of maximum thickness (Fig 1) and had normal signal intensity on both $\mathrm{T} 1$ and T2 images. The fornices were thickened; there were no malformations of cortical development. The directionally encoded color maps and diffusion tractography showed that the corpus callosum was bordered superiorly by longitudinal green fibers. The cingulum was fully formed and visually separable from the anomalous supracallosal fibers.

The second subject was a 20 -monthold girl initially referred at 11 months of age for new-onset febrile seizures and persistent lethargy. The patient had been born full-term after an uncomplicated pregnancy and was initially developmentally and physically normal, though developmental milestones were lagging at 20 months of age. Genetic analysis was not done. Assessed on the midsagittal T1 image, the corpus callosum was subjectively shortened; the rostral body measured $8.2 \mathrm{~mm}$ (Fig 2) with normal signal intensity on all sequences. There was restricted diffusion within the right mesial temporal lobe but no visible malformations of cortical development. Subsequent MR imaging at 20 months of age showed a stable appearance to the corpus callosum. There was interval development of right mesial temporal sclerosis with atrophy of the right fornix. The directionally encoded color map showed longitudinal midline supracallosal fibers in the thickened portion of the corpus callosum, which, by diffusion tractography, were separate from the cingulum.

The third subject was an 18-month-old boy referred for pervasive developmental delay, failure to thrive and recurrent aspiration. The patient had mild hemifacial microsomia, a malformed left thumb, and bilateral cryptorchidism. Findings of DNA microarray analysis by using comparative genomic hybridization were normal. MR imaging at $3 \mathrm{~T}$ showed that the callosal body measured $9.2 \mathrm{~mm}$ and was thicker than the splenium and genu (Fig 3) with normal signal intensity. There was bilateral peri-Sylvian and bifrontal polymicrogyria along with multifocal periventricular gray matter heterotopias. Diffusion imaging showed a triangle-shaped longitudinal midline supracallosal fiber bundle visually separate from the supracallosal portions of the cingulum, which appeared to fuse with the prefrontal segments of the cingulum.

The fourth subject was a 12-year-old boy with chronic seizure disorder and pervasive developmental delay with autism. There was mild facial dysmorphism and optic nerve pallor. Findings of genetic testing for Fragile X syndrome and microarray analysis by using a whole genome oligonucleotide array were normal, though analysis of the patient's DNA showed 3 copy losses (2q27.3, $3 \mathrm{p} 21.31,7 \mathrm{q} 21.11$ ) and 1 copy gain (8p11.23). MR imaging showed optic hypoplasia with preservation of the septum pellucidum. There was thickening of rostral callosal body (Fig 4), which measured $9.9 \mathrm{~mm}$; midline longitudinal supracallosal fibers were seen by diffusion imaging. The cingulum was difficult to identify on the color maps and by tractography.

\section{DISCUSSION}

Nonprogressive pervasive global developmental delay is relatively common, affecting $1 \%-3 \%$ of children. ${ }^{9}$ Along with routine cytogenetics and testing for Fragile $\mathrm{X}$ syndrome, MR imaging is suggested in the evaluation of developmental delay because MR imaging may show congenital malformations, brain injury, and unsuspected phakomatoses or metabolic disorders. ${ }^{9}$ DTI is a fairly recent addition to conventional MR imaging, which may provide additional information when cerebral malformations associated with the developmental delay involve larger white matter fibers. DTI can be acquired in routine clinical practice; a single acquisition that provides adequate SNR at $3 \mathrm{~T}$ adds approximately 4 minutes to the imaging time. Tensor data can be viewed as directionally encoded color maps or as 3D renderings by using a variety of readily available software tools. ${ }^{10}$ As the largest white matter bundle in the brain and by virtue of having a highly parallel structure, the corpus callosum is readily assessed with diffusion imaging. ${ }^{5}$ 

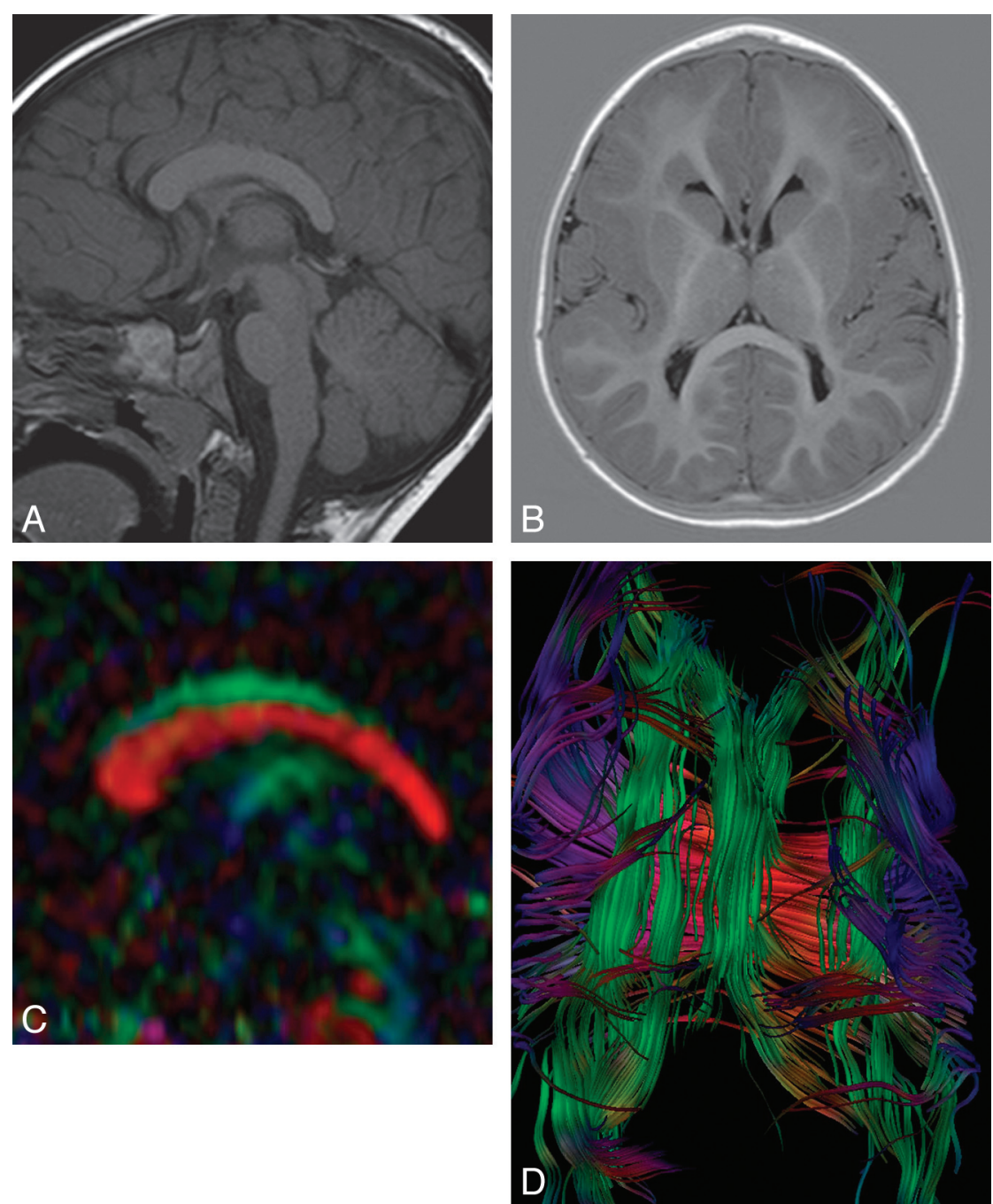

FIG 3. An 18-month-old boy referred for pervasive developmental delay, failure to thrive, and recurrent aspiration. $A$, Midsagittal Tl image shows that the rostral body is thicker $(9.9 \mathrm{~mm})$ than the splenium and genu. $B$, Axial $T 1$ inversion recovery image shows extensive frontal and periSylvian polymicrogyria and periventricular gray matter heterotopia. C, Sagittal color map shows longitudinal fibers in the midline above the corpus callosum. $D$, Axial top-down image from diffusion tractography shows the midline longitudinal fibers.

The normal corpus callosum is composed of transversely oriented red fibers that connect mirror-image regions of the cerebral hemispheres. ${ }^{4,5}$ In the normal brain, there are no midline longitudinal supracallosal fibers seen by diffusion imaging because all longitudinal fibers are, by definition, association fibers that connect heterotopic regions within the same hemisphere. The patients reported here are ones who underwent referral for variable developmental delay, in whom conventional MR imaging showed focal callosal thickening; additional insight into the malformed corpus callosum was provided by DTI, which showed that the callosal thickening was due to the presence of longitudinal midline supracallosal fibers. These fibers may represent the heterotopic cingulum, indusium griseum, or aberrantly oriented callosal axons.

The cingulum has longitudinally oriented parasagittal association fibers that form the outer limbic arch. ${ }^{11}$ Reports of anomalies of the cingulum are uncommon. Hori and $\operatorname{Stan}^{12}$ described 2 deceased adults with thick midline longitudinal midline fibers attributed to an aberrant cingulum on the basis of the attachment of the fibers to the cingulated gyrus. Friede and Briner ${ }^{13}$ described similar findings in a deceased patient who had undergone closure of a lumbar myelomeningocele at birth, but they speculated that the abnormal supracallosal tract was a malformation of the forniceal system rather than the cingulum. The cingulum is visually indistinguishable from regional white matter by conventional MR imaging but is readily identifiable by diffusion tractography as green fibers arching over the corpus callosum from the prefrontal to the retrosplenial region and extending into the temporal lobes. Diffusion tractography has been used to show anomalies of the cingulum and aberrant midline supracallosal fibers connected to the cingulum in patients with Chiari II malformations and in lobar holoprosencephaly. ${ }^{8,14}$

The IG is a supracallosal midline structure containing cells that produce the axonal guidance and repellant molecules critical to normal callosal formation. ${ }^{15}$ The IG is a small anatomic structure that may be visible in healthy adults by using high-resolution MR imaging as symmetric pairs of narrow gray matter strips, which may be centrally fused, or a single lateralized strip of neural tissue. ${ }^{16}$ The IG is presumed to be vestigial and nonfunctional in humans, though it may have some role in hippocampal seizure disorders because the IG is thought to have connections with hippocampal-amygdaloid pathways, the entorhinal cortex, dentate gyrus, medial septum, and piriform cortex. ${ }^{17} \mathrm{~A}$ small structure in the human brain, the IG is not commonly discussed in the imaging literature or noted in clinical practice. In knockout mice models, loss or significant reduction in the size of the IG is associated with failure of formation of the corpus callosum. ${ }^{17}$ Whether hypertrophy of the IG is associated with overgrowth of the corpus callosum is unknown.

The anomalous fibers that explain the segmental thickening of the corpus callosum may represent commissural axons that went astray during axonal migration. Aberrant migration of callosal axons have been reported in partial callosal agenesis; the longitudinal fiber bundle has been termed the "sigmoid aberrant bundle" by Wahl et al. ${ }^{4}$ They used high angular resolution diffusion imaging to study callosal connectivity in adults with partial callosal agenesis and described asymmetric aberrant bundles connecting the frontal lobe with the contralateral occipitoparietal cortex. ${ }^{4}$ The Probst bundles seen in callosal agenesis are also examples of misdirected callosal axons, though Probst bundles do not cross the midline. ${ }^{1,2}$ 

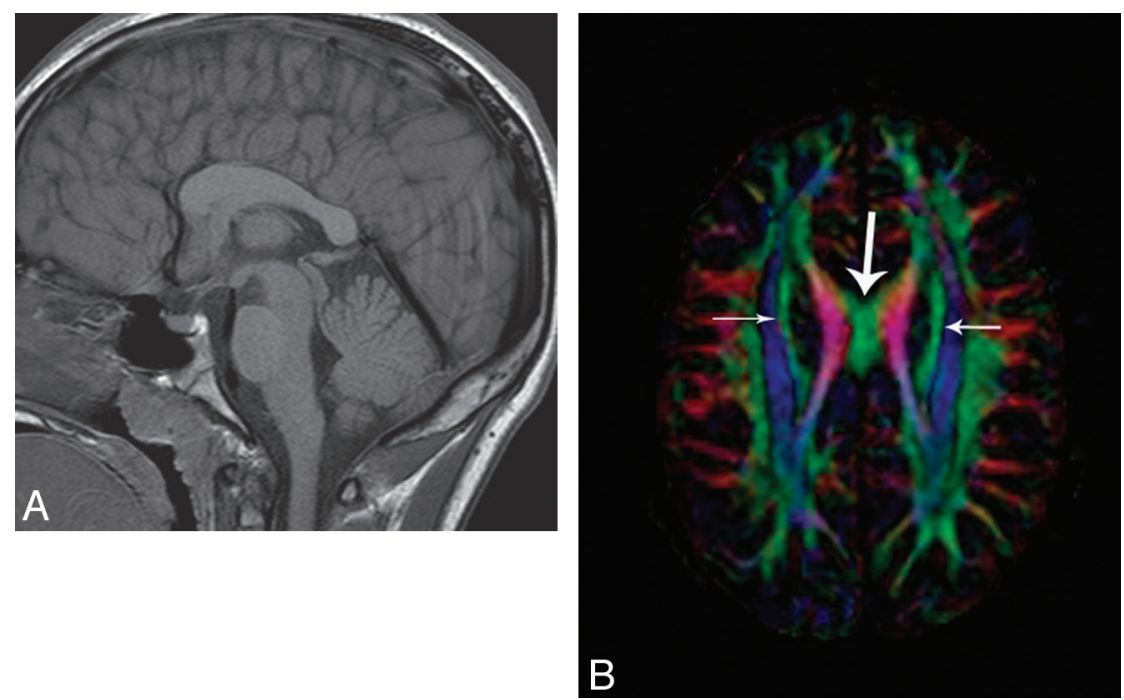

FIG 4. A 12-year-old boy with chronic seizure disorder and pervasive developmental delay with autism. $A$, On the midsagittal $\mathrm{T} 1$ image, the callosal length is normal. However, the rostral body is thicker than the splenium, measuring $9.2 \mathrm{~mm}$. There is optic hypoplasia. B, Axial color map shows a well-formed superior fronto-occipital (SFO) fasciculus (short arrows), and the anomalous midline fibers clearly are separable from the SFO fasciculus. In this patient, the cingulum was poorly formed.

The longitudinal fibers reported herein do not appear to represent the medially displaced superior occipitofrontal longitudinal fasciculus because this fiber bundle was identifiable in a normal location in all of the subjects. I do not believe the finding is artifacts because one would expect to have seen the artifacts in other cases during the past decade during which we have been doing diffusion tensor imaging, and we have not seen them. We have seen subjects with mild thickening of the rostral callosal body who did not demonstrate the anomalous supracallosal fibers; this finding suggests other explanations for congenital callosal thickening.

Congenital nonsyndromic thickening of the corpus callosum is rare. Lerman-Sagie et $\mathrm{al}^{7}$ reported 9 cases in which a thick corpus callosum was diagnosed on prenatal sonography; the malformation was associated with developmental delay and seizures in early childhood. Syndromic conditions associated with enlargement of the corpus callosum include NF type 1, Cohen syndrome, and M-CMTC. ${ }^{18-20}$ In NF type 1, the large commissural tracts may result from a lack of the normal postnatal apoptotic cell pruning documented in the young rhesus monkey and in mice. ${ }^{21,22}$ Cohen syndrome is characterized by failure to thrive, early-onset hypotonia, microcephaly, moderate-to-profound psychomotor retardation, and typical facial features. ${ }^{19} \mathrm{M}-\mathrm{CMTC}$ is a rare congenital syndrome of unknown etiology associated with enlargement of a portion or the entire corpus callosum. ${ }^{20}$ Neuroimaging in this rare disorder has shown cerebellar tonsillar herniation associated with rapid brain growth and progressive crowding of the posterior fossa during infancy along with focal cortical dysplasia, polymicrogyria primarily involving the periSylvian and insular regions, and cerebral and/or cerebellar asymmetric overgrowth. ${ }^{20}$ None of the 4 cases reported herein had other clinical or imaging stigmata of NF type 1, microcephaly, or evidence of M-CMTC, though 1 subject did have bifrontal and peri-Sylvian polymicrogyria.
Diffusion imaging can be used to quantify white matter differences in healthy and disease states and can provide information about fiber orientation and connectivity. ${ }^{10}$ The directional information about white matter fibers was used to create the color-coded FA maps and 3D mathematic depictions of the corpus callosum and supracallosal fibers. However, the use of diffusion tensor imaging precludes assessment of axonal connectivity due to the insensitivity of tensor imaging to intravoxel crossing fibers, and which regions of gray matter were connected by the anomalous supracallosal fibers or whether these fibers connected with the normally positioned cingulum could not be determined. The use of high-angulardiffusion imaging with multiple b-values would have been preferable for purposes of determining connectivity. However, because patient throughput limits the time allotted for imaging, we routinely limit diffusion imaging to diffusion tensor, notwithstanding the inherent limitations of DTI.

\section{CONCLUSIONS}

Diffusion imaging may provide insight into aberrant axonal migration associated with less common variants of callosal malformation, and diffusion imaging should be considered when children with global pervasive developmental delay show a malformation of larger white matter tracts by conventional MR imaging.

\section{REFERENCES}

1. Hetts SW, Sherr EH, Chao S, et al. Anomalies of the corpus callosum: An MR analysis of the phenotypic spectrum of associated malformations. AJR Am J Roentgenol 2006;187:1343-48

2. Paul LK, Brown WS, Adolphs R, et al. Agenesis of the corpus callosum: genetic development and functional aspects of connectivity. Nat Rev Neurosci 2007;8:287-99

3. Wahl M, Lauterbach-Soon B, Hattingen E, et al. Human motor corpus callosum: topography, somatotopy, and link between microstructure and function. J Neurosci 2007;27:12132-38

4. Wahl M, Strominger Z, Jeremy RJ, et al. Variability of homotopic and heterotopic callosal connectivity in partial agenesis of the corpus callosum: a 3T diffusion tensor imaging and Q-ball tractography study. AJNR Am J Neuroradiol 2009;30:282-89

5. Tovar-Moll F, Moll J, de Oliveira-Souza R, et al. Neuroplasticity in human callosal dysgenesis: a diffusion tensor imaging study. Cereb Cortex 2007;17:531-41

6. Rypens F, Sonigo P, Aubry MC, et al. Prenatal MR diagnosis of a thick corpus callosum. AJNR Am J Neuroradiol 1996;17:1918-20

7. Lerman-Sagie T, Ben-Sira L, Achiron R, et al. Thick fetal corpus callosum: an ominous sign? Ultrasound Obstet Gynecol 2009;34:55-61

8. Koob M, Weingertner AS, Gasser B, et al. Thick corpus callosum: a clue to the diagnosis of fetal septopreoptic holoprosencephaly? $P e-$ diatr Radiol 2012;42:886-90

9. Shevell M, Ashwal S, Donley D, et al. Practice parameter: evaluation of the child with global developmental delay: report of the Quality 
Standards Subcommittee of the American Academy of Neurology and the Practice Committee of the Child Neurology Society. Neurology 2003;60:367-80

10. Moldrich RX, Pannek K, Hoch R, et al. Comparative mouse brain tractography of diffusion magnetic resonance imaging. Neuroimage 2010;51:1027-36

11. Carpenter M, Sutin J. Core Text of Neuroanatomy. 3rd ed. Baltimore: Williams \& Wilkins; 1983

12. Hori A, Stan AC. Supracallosal longitudinal fiber bundle: heterotopic cingulum, dorsal fornix or Probst bundle? Neuropathology 2004;24:56-59

13. Friede RL and Briner J. Midline hyperplasia with malformation of the fornical system. Neurology 1978;28:1302-05

14. Vachha B, Adams RC, Rollins NK. Limbic tract anomalies in pediatric myelomeningocele and Chiari II malformation: anatomic correlations with memory and learning-initial investigation. Radiology 2006;240:194-202

15. Shu T, Li Y, Keller A, et al. The glial sling is a migratory population of developing neurons. Development 2003;130:2929-37

16. Nakada T. High-field, high-resolution MR imaging of the human indusium griseum. AJNR Am J Neuroradiol 1999;20:524-25
17. Wyss JM, Sripanidkulchai K. The indusium griseum and anterior hippocampal continuation in the rat. J Comp Neurol 1983;20;219:251-72

18. Dubovsky EC, Booth TN, Vezina G, et al. MR imaging of the corpus callosum in pediatric patients with neurofibromatosis type 1. AJNR Am J Neuroradiol 2001;22:190-95

19. Kivitie-Kallio S, Autti T, Salonen O, et al. MRI of the brain in the Cohen syndrome: a relatively large corpus callosum in patients with mental retardation and microcephaly. Neuropediatrics 1998;29:298-301

20. Conway RL, Pressman BD, Dobyns WB, et al. Neuroimaging findings in macrocephaly-capillary malformation: a longitudinal study of 17 patients. Am J Med Genet A 2007;143A:2981-3008

21. LaMantia AS, Rakic P. Axon overproduction and elimination in the corpus callosum of the developing rhesus monkey. J Neurosci 1990;10:2156-75

22. Siman R, Bozyczko-Coyne D, Meyer SL, et al. Immunolocalization of caspase proteolysis in situ: evidence for widespread caspase-mediated apoptosis of neurons and glia in the postnatal rat brain. $\mathrm{Neu}$ roscience 1999;92:1425-42 